Article

\title{
Dark Solitons and Grey Solitons in Waveguide Arrays with Long-Range Linear Coupling Effects
}

\author{
Zhijie Mai ${ }^{1}$, Haitao $\mathrm{Xu}^{1}{ }^{1}$, Fang Lin ${ }^{1}$, Yan Liu ${ }^{1, *}$, Shenhe $\mathrm{Fu}^{2}$ and Yongyao $\mathrm{Li}^{3}$ \\ 1 Department of Applied Physics, South China Agricultural University, Guangzhou 510642, China; \\ mhero14@163.com (Z.M.); xuhaitao@scau.edu.cn (H.X.); flin_163@163.com (F.L.) \\ 2 Department of Optoelectronic Engineering, Jinan University, Guangzhou 510632, China; \\ fushenhe@jnu.edu.cn \\ 3 School of Physics and Optoelectronic Engineering, Foshan University, Foshan 528000, China; \\ yongyaoli@gmail.com \\ * Correspondence: lycalm@scau.edu.cn; Tel.: +86-137-6064-7286
}

Academic Editors: Boris Malomed and Christophe Finot

Received: 23 December 2016; Accepted: 17 March 2017; Published: 22 March 2017

\begin{abstract}
In J. Phys. Soc. Jpn. 83, 034404 (2014), we designed a scheme of waveguide arrays with long-range linear coupling effects and studied the bright solitons in this system. In this paper, we further study the dynamics of dark and grey solitons in such waveguide arrays. The numerical simulations show that the stabilities of dark solitons and grey solitons depend on the normalized decay length and the scaled input power. The width of dark solitons and the grey level of grey solitons are studied. Our results may contribute to the understanding of discrete solitons in long-range linear coupling waveguide arrays, and may have potential applications in optical communications and all-optical networks.
\end{abstract}

Keywords: discrete solitons; dark solitons; grey solitons; long-range coupling effects; waveguide arrays

\section{Introduction}

Nonlinear discrete systems attract considerable attention in many branches of physics, and exhibit various physical characteristics [1-3]. In the fields of optics [4-6] and Bose-Einstein condensates (BECs) [7-9], the evolution of nonlinear waves in discrete systems is a popular topic.

The basic model of a nonlinear discrete system in optics is an array of evanescently-coupled waveguides consisting of nonlinear materials. In a waveguide array, the propagation of light is primarily characterized by the coupling caused by the overlap between the fundamental modes of nearest-neighbouring waveguides. A crucial issue is to study the formation and properties of discrete solitons (DSs) in such nonlinear waveguide systems. DS formation is the result of a balance between on-site nonlinearity and the discrete diffraction induced by linear coupling among adjacent waveguides or lattice sites. DSs show strong potential for application in all-optical data processing; their most attractive feature is that DSs can enable intelligent functional operations such as routing, blocking, logic functions, and time gating in many all-optical devices [10].

An interesting extension is to investigate the formation of solitons in the presence of nonlocal effects. Trillo and colleagues studied the shock waves and dark solitons in nonlocal nonlinear media [11,12]. A next-nearest neighbour (NNN) model in which the linear coupling matrix becomes a quadruple-diagonal matrix after higher-order diffraction was studied by Kevrekidis and colleagues [13]. In 2012, Noskov and colleagues conducted significant studies of the nonlinear dipolar field in a nanoparticle train [14-16] — which can be viewed as a discrete nonlinear system-and reported that a linear coupling effect can exist among all lattice sites because of long-range dipole-dipole interactions. This system can produce all non-zero off-diagonal elements in the linear coupling matrix. 
Long-range coupling in waveguide arrays is the coupling between the waveguides that were spaced with certain distance-it can affect the propagation dynamics of light field. Long-range coupling is different from that of the conventional waveguide arrays, which only consider the coupling between the adjacent waveguides (i.e., the short-range coupling). We have designed such a waveguide array with long-range coupling and studied the formation of bright solitons-see our previous paper in [17]. In this work, we further study the dynamics of dark and grey solitons in such a waveguide array, which was not considered before. The numerical results show that: regarding the dark solitons, the stability and width strongly depend on the mean power and the normalized decay length, which describes the effective length of the coupling effects in the waveguides; regarding the grey solitons, the stability and grey level are determined by the mean power and the normalized decay length.

The remainder of this paper is organized as follows. We provide a brief description of the model and basic equations in Section 2. Then, we study dark solitons in Section 3 and the grey solitons in Section 4. The paper is concluded in Section 5.

\section{Model and Basic Equations}

In [17], we designed a scheme for an optics experiment to apply our model of long-range linear coupling waveguide arrays. The model is built based on an AlGaAs single-mode waveguide structure. The real-scale linear coupling parameter between different waveguides can be fitted with an exponential decay, hence the long-range linear coupling effect is introduced in the system.

In our scheme, DSs can be described by the following equation, which is adapted from the discrete nonlinear Schrödinger equation [18,19]:

$$
i \frac{\partial}{\partial z} u_{n}=\gamma\left|u_{n}\right|^{2} u_{n}-\sum_{m} C_{m n} u_{m}
$$

Here, $\gamma$ is the fixed nonlinear parameter of the system, where $\gamma=1$ or $\gamma=-1$ indicates that the system features self-focusing or self-defocusing nonlinearity, respectively. $u_{n}$ is the field amplitude of the $n$-th mode. Because each waveguide is identical, for simplicity, the propagation constant $\beta$ is absorbed into the phase of $u_{n} . z$ is the propagation distance along the waveguides, $n$ is the number of waveguides, and the coefficient $C_{m n}$ defines the coupling, which depends on the optical wavelength and the field overlap between $\mathrm{m}$-th waveguide and n-th waveguide. Generally, Equation (1) can be expressed in matrix form as follows:

$$
i \frac{\partial}{\partial z} U=(C+V) U
$$

where the matrix $U$ and the elements of the matrix $V$ are defined as $U=\left(u_{1}, \cdots, u_{N}\right)^{T}$ (where the superscript $\mathrm{T}$ indicates the transposition of the matrix and $N$ is the number of waveguides) and $V_{m n}=\gamma\left|u_{m}\right|^{2} \delta_{m n}$ (where $\delta_{m n}$ is the Kronecker symbol), respectively.

The total power of the guide mode in the system is given by $P=\sum_{n}^{N}\left|u_{n}\right|^{2}$. In the model, we consider the matrix elements $C_{m n}$ in Equation (1) to be given by

$$
C_{m n}= \begin{cases}c_{0} \exp (-j / d) & (j \neq-1), \\ 0 & (j=-1),\end{cases}
$$

where $c_{0}$ is the control parameter, $j=|m-n|-1$, and $d$ is the normalized decay length, which describes the effective length of the coupling effects in the waveguides. When $d \ll 1$, the system corresponds to a nearest-neighbour-coupled model. By contrast, when $d \gg 1$, the system exhibits strong coupling effects. Equation (3) forms the linear coupling matrix $C$, which represents the long-range linear 
coupling interactions among all lattice sites. In optics, when the separation between waveguides is sufficiently narrow, such higher-order cross-coupling effects can be induced [20].

The relationship between the scaled parameters in Equation (1) and the real-scale parameters are given in [17] in detail.

We assume that the soliton solutions for Equation (1) are written as

$$
u_{n}(z)=\phi_{n} e^{-i \mu z},
$$

where $\phi_{n}$ is the stationary solution and $-\mu$ is the propagation constant, which is defined as

$$
\mu=\frac{U^{\dagger}(C+V) U}{P} .
$$

$U$ is the solution of field amplitude in matrix form and $U^{+}$is the conjugated matrix of $U$. The stability of stationary solitons can be numerically determined by computing the eigenvalues for small perturbations or through direct simulations. The perturbed solution is given as $u_{n}=e^{-i \mu z}\left(\phi_{n}+w_{n} e^{i \lambda z}+v_{n}^{*} e^{-i \lambda^{*} z}\right)$. Substituting this solution into Equation (1) and linearizing yields the following eigenvalue problem:

$$
\left(\begin{array}{cc}
C-\mu+2 V & \Phi \\
-\Phi^{*} & -C+\mu-2 V
\end{array}\right)\left(\begin{array}{c}
w \\
v
\end{array}\right)=\lambda\left(\begin{array}{c}
w \\
v
\end{array}\right)
$$

where the elements of the matrix $\Phi$ are defined as $\Phi_{m n}=\gamma \phi_{m}^{2} \delta_{m n}$.

The solution $\phi$ is stable if all eigenvalues $\lambda$ are real.

\section{Dark Solitons}

In numerical simulations, we apply the imaginary time propagation (ITP) method [21] to study the fundamental solution to Equation (1) for dark solitons. We find that the stability of the dark soliton solutions changes with the scaled total power $P$ and the normalized decay length $d$. When the eigenvalues $\lambda$ have an imaginary part, this means that the solution of $\phi$ is unstable. Figure $1 \mathrm{a}, \mathrm{b}$ show that the value of the imaginary part of $\lambda(\operatorname{Im}(\lambda))$ varies with $P$ and $d$. From Figure 1 a, we can see two traits of dark solitons in this long-range linear coupling waveguide array. First, the nonlinearity strengthens when $P$ increases, and the system requires a stronger coupling effect to achieve a stable solution for dark solitons. It can be seen that $d$ increases as $P$ increases. For example, when $P=0.5$ and $d>1.5$, the solitons are stable $(\operatorname{Im}(\lambda)=0)$; however, when $P$ increases to $P=1$, the coupling effect must be enhanced to $d>2.2$ to achieve stable solitons. Second, when the effective length $d$ of the coupling effect is fixed, $\operatorname{Im}(\lambda)$ increases with larger $P$. This means that the instability is enhanced when the scaled total power $P$ is higher. From Figure $1 b$, we can see when the system has either a strongly local effect $(d=0.0001)$ or a strongly coupling effect $(d=5)$; the dark soliton solution is stable regardless of the value of $P$. However, for $0.0001<d<5$, the system is subject to the combined action of the short-range and long-range effects, and the stability conditions for dark solitons are more complex. In this region of $d$, the tendency is that dark solitons become more unstable as $P$ increases. Figure $1 \mathrm{c}, \mathrm{d}$ show the amplitude and intensity, respectively, of the fundamental solution for dark solitons with $P=1$ and $d=3$. In Figure 1e, we present an example of the evolution of stable dark solitons with $P=1$ and $d=3$. In Figure 1f, we present an example of the evolution of unstable dark solitons with $P=0.5$ and $d=0.8$.

We also present the widths of dark solitons in this system for different $d$ and $P$ values, as shown in Figure 2. The dotted lines represent unstable dark soliton solutions (with $\operatorname{Im}(\lambda) \neq 0$ ), and the solid lines represent stable solutions. 


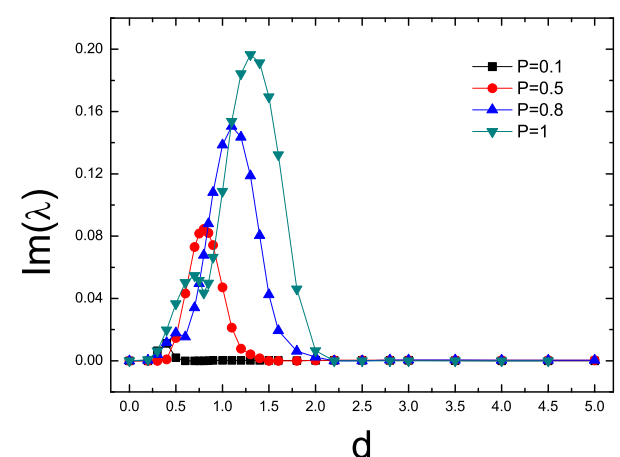

(a)

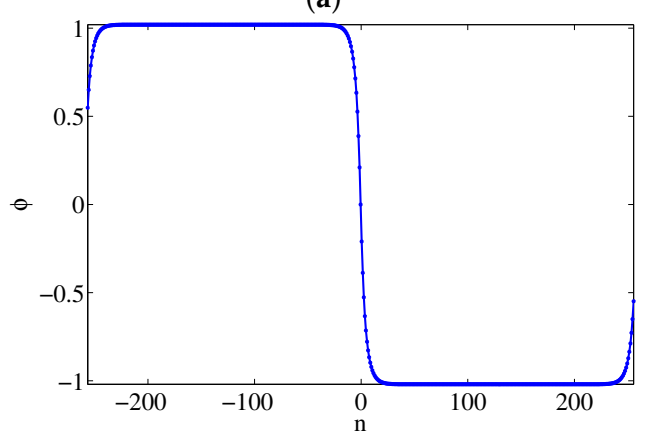

(c)

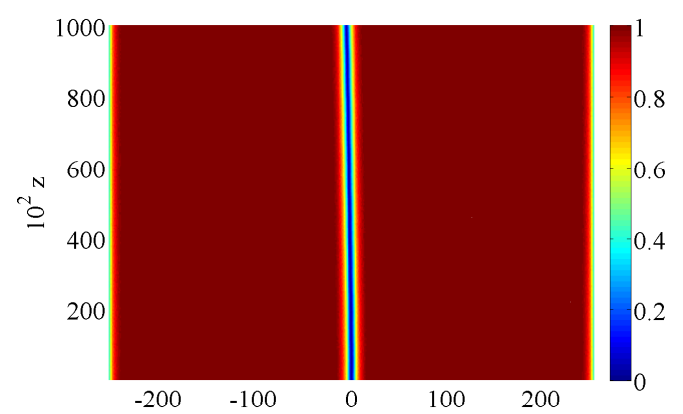

(e)

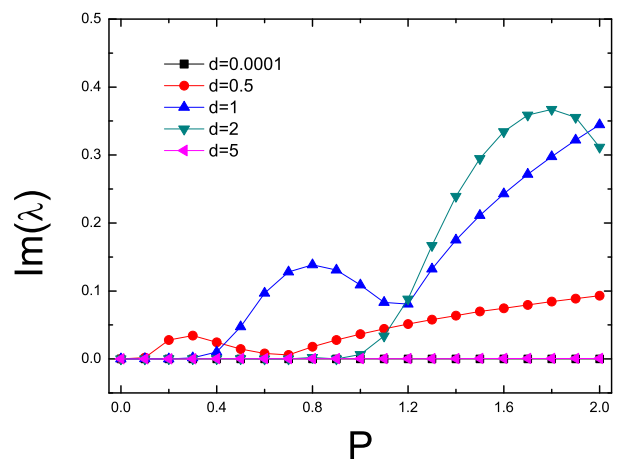

(b)

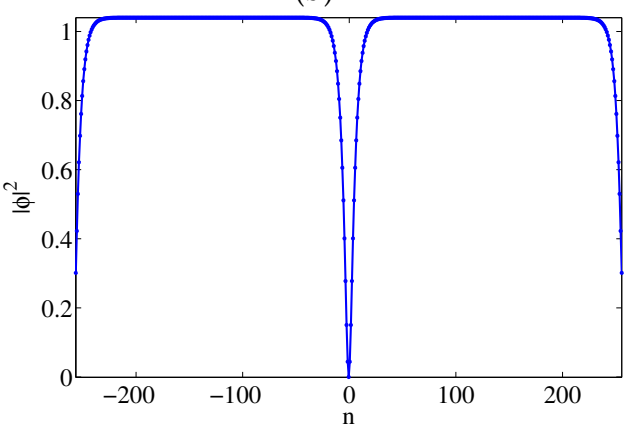

(d)

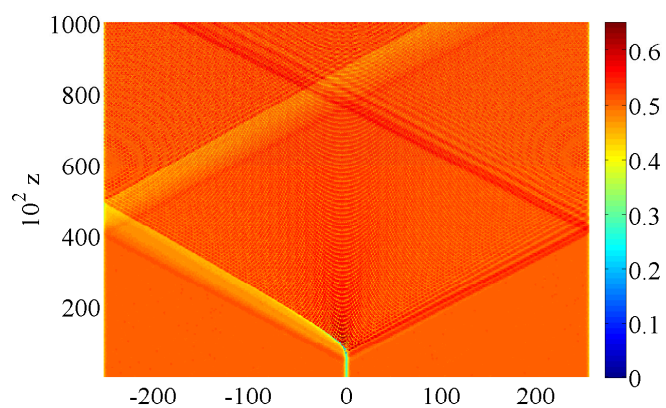

(f)

Figure 1. Dark solitons in a 513-waveguide array. (a) The imaginary part of the eigenvalues $\lambda(\operatorname{Im}(\lambda))$ for different $d$ values; (b) The imaginary part of the eigenvalues $\lambda(\operatorname{Im}(\lambda))$ for different $P$ values; (c) The amplitude of the fundamental solution for dark solitons $(P=1$ and $d=3)$; (d) The intensity of the fundamental solution for dark solitons ( $P=1$ and $d=3$ ); (e) The evolution of a stable solution for dark solitons $(P=1$ and $d=3)$; (f) The evolution of an unstable solution for dark solitons $(P=0.5$ and $d=0.8)$.

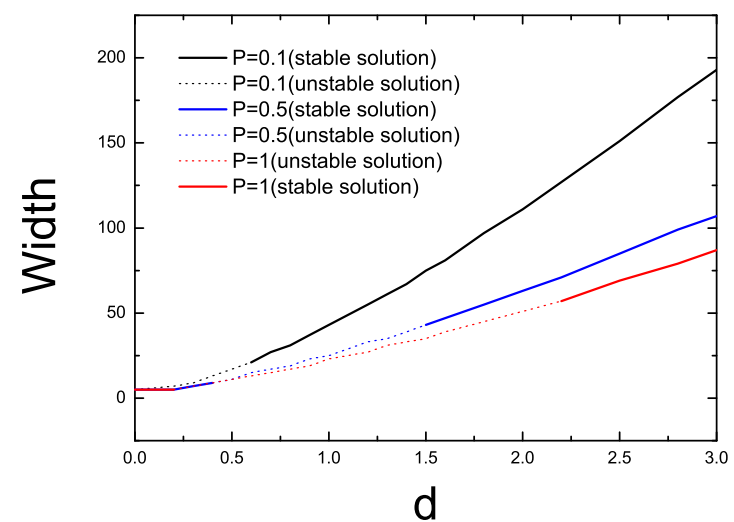

Figure 2. Widths of dark solitons for different $d$ and $P$ values. 


\section{Grey Solitons}

We use the same method (ITP) to study the fundamental solution for grey solitons. Figure 3 shows the characteristics of grey solitons. Figure $3 a, b$ show that the value of the imaginary part of $\lambda(\operatorname{Im}(\lambda))$ varies with $P$ and $d$. Grey solitons in this long-range linear coupling system have features similar to those of dark solitons: $d$ increases as $P$ increases (Figure 3a); when the effective length $d$ of the coupling effect is fixed, $\operatorname{Im}(\lambda)$ increases with higher $P$ (Figure 3a), and grey solitons become more unstable as $P$ increases in the region of $0.0001<d<5$ (Figure $3 \mathrm{~b}$ ). However, grey solitons also show some distinct features compared with dark solitons; for example, the instability of grey solitons is stronger than that of dark solitons. First, by comparing Figure 3a,b with Figure $1 \mathrm{a}, \mathrm{b}$, we can see that $\operatorname{Im}(\lambda)$ is larger for grey solitons than for dark solitons given the same $P$ and $d$. Second, the threshold in $d$ where solitons transition from instability to stability lies at a higher value for grey solitons than for dark solitons. For example, as seen in Figure 1a, the threshold is $d=2.2$ for $P=1$, whereas in Figure 3a, the threshold is $d=3$ for $P=1$. Figure $3 \mathrm{c}$, d show the amplitude and intensity, respectively, of the fundamental solution for grey solitons with $P=1$ and $d=3$. In Figure 3e, we present an example of the evolution of stable grey solitons with $P=1$ and $d=3$. In Figure 3f, we present an example of the evolution of unstable grey solitons with $P=0.5$ and $d=0.8$.

We also plot the grey levels for grey solitons with different $d$ and $P$ values, as shown in Figure 4 . The grey level is defined as follows:

$$
\text { Greylevel }=\frac{\left|\max \left(u_{n}\right)\right|^{2}}{\left|\min \left(u_{n}\right)\right|^{2}} .
$$

The dotted lines represent unstable grey soliton solutions (with $\operatorname{Im}(\lambda) \neq 0$ ), and the solid lines represent stable solutions.

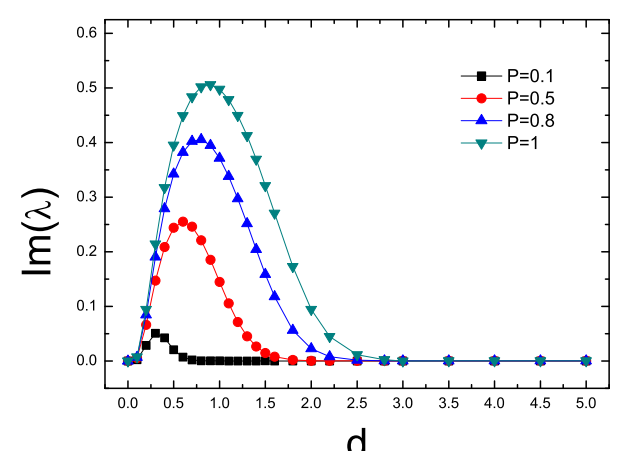

d

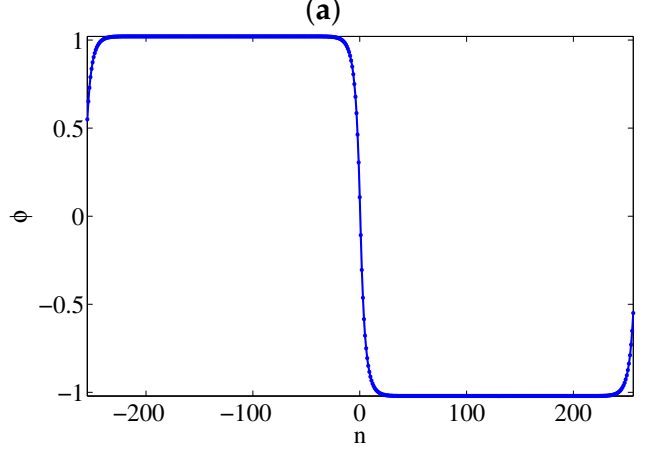

(c)

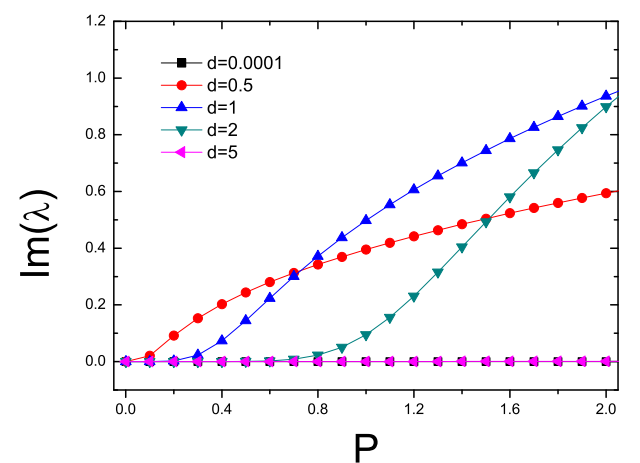

(b)

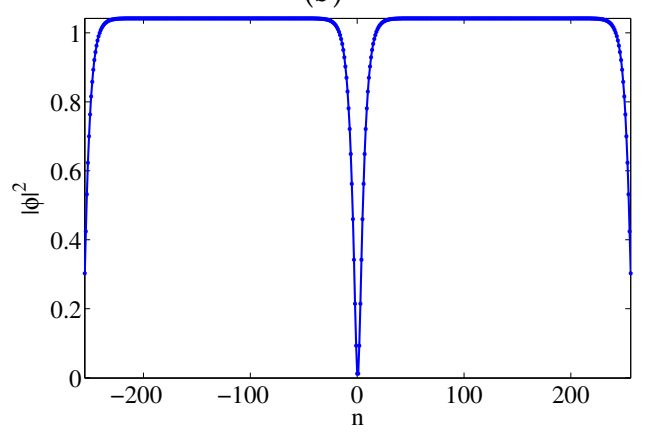

(d)

Figure 3. Cont. 


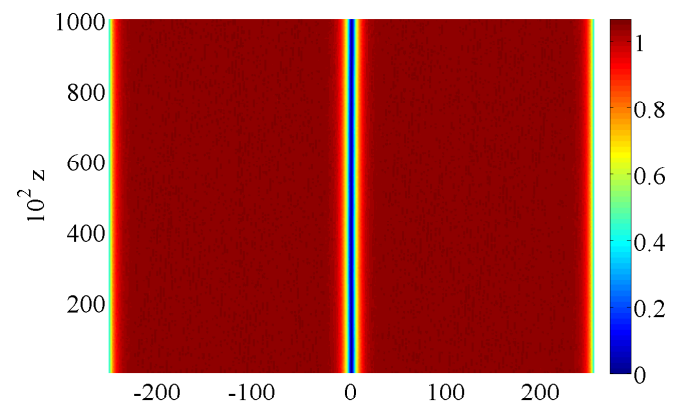

(e)

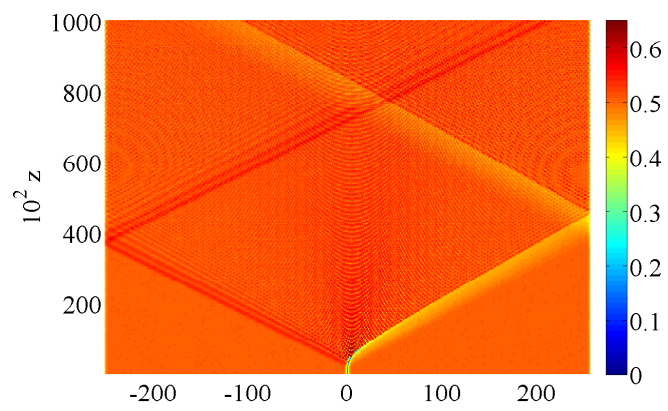

(f)

Figure 3. Grey solitons in a 512-waveguide array. (a) The imaginary part of the eigenvalues $\lambda(\operatorname{Im}(\lambda))$ for different $d$ values; (b) The imaginary part of the eigenvalues $\lambda(\operatorname{Im}(\lambda))$ for different $P$ values; (c) The amplitude of the fundamental solution for grey solitons $(P=1$ and $d=3)$; (d) The intensity of the fundamental solution for grey solitons $(P=1$ and $d=3)$; (e) The evolution of a stable solution for grey solitons $(P=1$ and $d=3)$; (f) The evolution of an unstable solution for grey solitons $(P=0.5$ and $d=0.8)$.

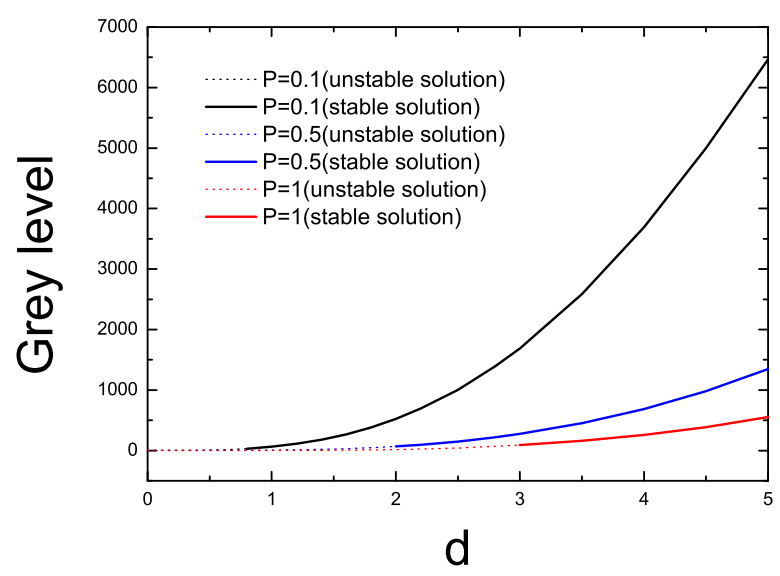

Figure 4. Grey levels of grey solitons with different $d$ and $P$ values.

\section{Conclusions}

In conclusion, we performed numerical studies of dark solitons and grey solitons in a waveguide array with long-range linear coupling effect. This system is described by the discrete nonlinear Schrödinger equation with off-diagonal elements in the linear coupling matrix filled with non-zero interaction terms. The stabilities of dark solitons and grey solitons are studied. The features of solitons such as the widths of dark solitons and the grey levels of grey solitons are comprehensively studied. Our results may fill the gap in the understanding of discrete solitons in long-range linear coupling waveguide arrays, and our design may have potential applications in optical communications and all-optical networks.

Acknowledgments: This work was supported, in part, by the National Natural Science Foundation of China through Grant Nos. 61571197, 11575063, and 61172011.

Author Contributions: Zhijie Mai, Yan Liu and Yongyao Li conceived of and designed the model. Zhijie Mai performed the numerical simulations. Haitao Xu and Fang Lin analysed the data. Shenhe Fu and Yongyao Li provided theoretical explanations. Zhijie Mai and Yan Liu wrote the paper.

Conflicts of Interest: The founding sponsors had no role in the design of the study; in the collection, analysis, or interpretation of the data; in the writing of the manuscript; or in the decision to publish the results. 


\section{References}

1. Lederer, F.; Stegemanb, G.I.; Christodoulides, D.N.; Assanto, G.; Segev, M.; Silberberg, Y. Discrete solitons in optics. Phys. Rep. 2008, 463, 1-126.

2. Flach, S.; Gorbachb, A.V. Discrete breathers-Advances in theory and applications. Phys. Rep. 2008, 467, $1-116$.

3. Kartashov, Y.V.; Malomed, B.A.; Torner, L. Solitons in nonlinear lattices. Rev. Mod. Phys. 2011, 83, 247-306.

4. Christodoulides, D.N.; Lederer, F.; Silberberg, Y. Discretizing light behaviour in linear and nonlinear waveguide lattices. Nature 2003, 424, 817-823.

5. Garanovich, I.L.; Longhi, S.; Sukhorukova, A.A.; Kivshar, Y.S. Light propagation and localization in modulated photonic lattices and waveguides. Phys. Rep. 2012, 518, 1-79.

6. Chen, Z.; Segev, M. Christodoulides, D.N. Optical spatial solitons: Historical overview and recent advances. Rep. Prog. Phys. 2012, 75, 086401.

7. Trombettoni, A.; Smerzi, A. Discrete solitons and breathers with dilute Bose-Einstein condensates. Phys. Rev. Lett. 2001, 86, 2353-2356.

8. Efremidis, N.K.; Christodoulides, D.N. Lattice solitons in Bose-Einstein condensates. Phys. Rev. A 2003, $67,063608$.

9. Morsch, O.; Oberthaler, M. Dynamics of Bose-Einstein condensates in optical lattices. Rev. Mod. Phys. 2006, 78, 179-215.

10. Christodoulides, D.N.; Eugenieva, E.D. Blocking and routing discrete solitons in two-dimensional networks of nonlinear waveguide arrays. Phys. Rev. Lett. 2001, 87, 160-161.

11. Ghofraniha, N.; Conti. C.; Ruocco, G.; Trillo, S. Shocks in nonlocal media. Phys. Rev. Lett. 2007, $99,043903$.

12. Armaroli, A.; Fratalocchi, A.; Trillo, S. Suppression of transverse instabilities of dark solitons and their dispersive shock waves. Phys. Rev. A 2012, 80, 72.

13. Kevrekidis, P.G.; Malomed, B.A.; Saxena, A.; Bishop, A.R.; Frantzeskakis, D.J. Higher-order lattice diffraction: Solitons in the discrete NLS equation with next-nearest-neighbor interactions. Physica D 2003, 183, 87-101.

14. Noskov, R.E.; Belov, P.A.; Kivshar, Y.S. Subwavelength modulational instability and plasmon oscillons in nanoparticle arrays. Phys. Rev. Lett. 2012, 108, 324-329.

15. Noskov, R.E.; Belov, P.A.; Kivshar, Y.S. Oscillons, soltions, and domain walls in arrays of nonlinear plasmonic nanoparticles. Sci. Rep. 2012, 2, 873.

16. Noskov, R.E.; Belov, P.A.; Kivshar, Y.S. Subwavelength plasmonic kinks in arrays of metallic nanoparticles. Opt. Exp. 2012, 20, 2733-2739.

17. Mai, Z.; Fu, S.; Wu, J.; Li, Y. Discrete solitons in waveguide arrays with long-range linearly coupled effect. J. Phys. Soc. Jpn. 2014, 83, 034404.

18. Christodoulides, D.N.; Joseph, R.I. Discrete self-focusing in nonlinear arrays of coupled waveguides. Opt. Lett. 1998, 13, 794-796;

19. Eisenberg, H.S.; Silberberg, Y.; Morandotti, R.; Boyd, A.R.; Aitchison, J.S. Discrete Spatial Optical Solitons in Waveguide Arrays. Phys. Rev. Lett. 1998, 81, 3383-3386.

20. Gordon, R. Harmonic oscillation in a spatially finite array waveguide. Opt. Lett. 2004, 29, 2752-2754.

21. Chiofalo, M.L.; Succi, S.; Tosi, M.P. Ground state of trapped interacting bose-einstein condensates by an explicit imaginary-time algorithm. Phys. Rev. E 2000, 62, 7438-7444.

(C) 2017 by the authors. Licensee MDPI, Basel, Switzerland. This article is an open access article distributed under the terms and conditions of the Creative Commons Attribution (CC BY) license (http://creativecommons.org/licenses/by/4.0/). 